\title{
Between the Lines: Measuring Areal Displacement in Line Simplification
}

\author{
Barry J Kronenfeld ${ }^{\mathrm{a},}$, Jiaxin Deng ${ }^{\mathrm{a}}$ \\ ${ }^{a}$ Eastern Illinois University, bjkronenfeld@eiu.edu, jdeng@eiu.edu \\ * Corresponding author
}

\begin{abstract}
Quantitative measures of error are needed to complement subjective characterization of shape characteristics in the assessment of line simplication algorithms. Areal displacement is one of six metrics recommended for this purpose by McMaster in 1986. However, previous cartographers have failed to notice semantic ambiguities that obfuscate its meaning. This paper discusses semantic and computational aspects of areal displacement. Three distinct semantic definitions are identified. A simple definition derived from topological enclosure is shown to produce unintuitive results in certain regularly encountered situations. A more intuitively valid measure of areal displacement as a dynamic process is captured by the topological concept of minimum homotopy area, but robust, practical and efficient computation remains an active area of research. A third definition, referred to as shift displacement, is proposed that derives from the perspective of external regions that "shift sides" during the transformation of a line to its simplified form. A simple yet robust and computationally efficient algorithm is presented for computing displacement under the proposed definition.
\end{abstract}

Keywords: cartographic generalization, line simplification, areal displacement, shoelace formula

\section{Introduction}

Procedures to simplify lines and polygons play an important role in cartographic production and data dissemination. To evaluate alternative line simplification procedures, standardized error metrics are needed that describe the degree to which a simplified line diverges geometrically from the original line from which it was derived. Similar measures of displacement are required for assessing the positional accuracy of one line with respect to another reference line (). One natural class of metrics that has been proposed is areal displacement (McMaster 1986), which may loosely be described as the area separating the original and simplified line. Areal displacement has been used by several authors to quantify error in line simplification algorithms (White 1985, Visvalingam and Whyatt 1990, Shen et al 2018, Stanislawski et al. 2018), and has been observed to provide a good characterization of shape retention in the early (i.e. "weeding") stages of simplification (Muller 1987, Visvalingam and Whyatt 1990). It is also the objective function minimized locally at each step in the widely used algorithm of Visvalingam and Whyatt (1993).

It is possible that most authors assume measurement of areal displacement to be uncomplicated. McMaster describes computation simply as a matter of summing the "areal... difference between the base line and simplification" (McMaster 1986, p. 112). Other authors similarly describe measuring the area of "error polygons" (White 1985, p. 21) or else simply refer to MacMaster's 1986 paper as authoritative (Visavalingam and Whyatt
1990, Shen et al. 2018). On the other hand, there exist certain ambiguities in the definition of areal displacement that are well-known in mathematical topology (Chambers and Vejdemo-Johansson 2015). Although these ambiguities were not mentioned by earlier cartographic researchers, our experience suggests that they are unlikely to have gone entirely unnoticed by them, and instead we suspect that the problems they caused were handled individually on a case-by-case basis using "common sense" fixes. Such an approach, however, risks inconsistency and systematic bias.

To illustrate the problem to the skeptical reader and thus motivate the remainder of the paper, a scenario is presented in which naïve measurement of areal displacement as the simple sum of areas of enclosed polygons produces a rather unintuitive and misleading result (Figure 1).

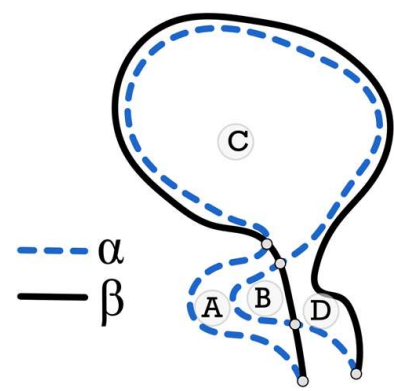

Figure 1. A simple scenario in which measuring areal displacement as the sum of areas of enclosed polygons leads to unintuitive results. 
In this and remaining figures, the original polyline $(\alpha)$ is shown as a dashed blue line and the simplified polyline $(\beta)$ as a solid black line. The polylines in Figure 1 start and end at the same locations and intersect each other at three other points. The total area of the enclosed polygons is $\mathrm{A}+\mathrm{B}+\mathrm{C}+\mathrm{D}$ (for simplicity, hereafter a capital letter is used to refer to both a simple enclosed polygon and its area). Intuitively, however, it does not seem right to include the large central polygon $\mathrm{C}$ in a measure of areal displacement. For one thing, this region seems to be within a large bend of both polylines, in the same general position with respect to each one and therefore not "displaced". Furthermore, no part of either polyline needs to pass through this region in order to be transformed into the other; to bring the two lines into concordance, one can imagine pulling the lower left-protruding bends in $\alpha$ to the right across $\mathrm{A}, \mathrm{B} \& \mathrm{D}$ until they match $\beta$ exactly, without ever passing through polygon $\mathrm{C}$.

Indeed, it will be shown that at least three different definitions of areal displacement are possible, each reasonable and intuitive. What's more, the three definitions lead to different values for the scenario depicted in Figure 1, as well as for other well-defined scenarios. On the other hand, they lead to exactly the same value for a simpler "standard" scenario, and thus their distinction is likely to be missed by the casual observer. This paper aims to expose the cartographic community to these different possible interpretations of areal displacement and to discuss issues involved in their definition, computation and interpretation.

The paper begins by describing four commonly encountered types of crossing patterns that can exist between two polylines that share the same start and end points. Three alternative measures of areal displacement are then defined, their differences are illustrated for each crossing pattern, and their computation is discussed. The third measure, shift dispacement, is novel as far as we are aware, certainly in its application to cartographic generalization although the underlying concepts are well known in the mathematical topology community. Shift displacement represents an alternative to existing metrics that balances the need for practical, efficient computation with semantic plausibility, for example avoiding the undesirable result described above for Figure 1. The final section discusses differences between metrics and avenues for future research.

Throughout, we assume that the original and simplified polylines share the same start and end points. This is guaranteed with most existing line simplification algorithms.

\section{Crossing Patterns}

When considering the concordance of two polylines $\alpha$ and $\beta$, it is useful to begin by enumerating their intersection points. The sequence and topology of these intersections, their adjacent line segments and enclosed regions yields a typology of crossing patterns, illustrated in Figure 2.

A standard crossing pattern (Figure 2a) may be defined as one in which each polyline traverses the intersection points in the same sequence and each line segment runs along the unbounded exterior region. In such a pattern, enclosed polygons will alternate to the right and left of each polyline, touching only at their intersection points (e.g. point b in Figure 2a).

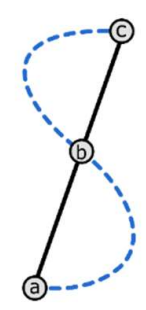

(a)

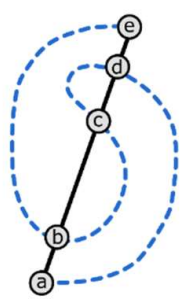

(b)

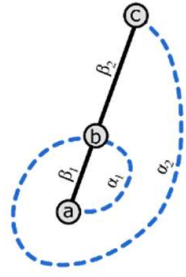

(c)

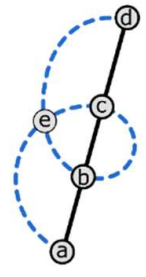

(d)
Figure 2: Crossing patterns between two polylines sharing the same start and end points. (a) standard, (b) back-crossing, (c) enclosed endpoint, and (d) self-intersection.

A non-standard crossing pattern consists of at least one of three elements. A back-crossing (Figure 2b) occurs when one polyline traverses the intersections in a different sequence than the other. For example, in Figure $2 b$ the intersections are traversed in sequence [adcbe] along $\alpha$, but in sequence [abcde] along $\beta$. An enclosed endpoint (Figure 2c) occurs when either the shared start or end point is fully enclosed by any set of line segments. For example, in Figure 2c the shared start point $\mathbf{a}$ of both lines is fully enclosed by line segments $\alpha_{2}$ and $\beta_{2}$. Lastly, a self-intersection (e.g. point $\mathbf{e}$ in Figure 2d) occurs when either polyline crosses itself. Note that any combination of back-crossings, enclosed endpoints and selfintersections may occur, resulting in a variety of complex topological configurations.

Non-standard patterns are likely to occur at least occasionally as the result of any line simplification algorithm. Back-crossings will occur when a sinuous feature with a "double-meander" (such as the dashed line in Figure 2b) is simplified to a sufficient degree, as extreme simplification will eventually result in a straight line. For an enclosed endpoint to occur, it is only necessary that a large spiraling meander appears near the beginning or end of the feature. Finally, self-intersections often occur as an unintended artifact of line simplification, and while various algorithm enhancements and post-processing methods have been developed to avoid or remove self-intersections (e.g. Saalfeld 1999, Lee and Hardy 2005, Raposo 2013), they can be computationally expensive and are not universally implemented. Although many consider self-intersections to be unacceptable in any cartographic process, we include them in our analysis for completeness.

The effects of non-standard patterns can be large even when they involve only small portions of the polyline. This is especially true when back-crossings and selfintersections occur at narrow gaps between sequentially distant portions of the polyline. For example, the situation depicted in Figure 1 is topologically equivalent to Figure $2 \mathrm{~b}$, but the effect is more pronounced due to the geometry 
of the polyline. Similar examples frequently occur due to self-intersections.

\section{Alternate Definitions of Areal Displacement}

We now introduce three different ways of defining areal displacement, each of which seems natural and intuitive (Figure 3). Displacement area may be defined simply as the total area of all regions in the plane that are completely enclosed by sections of $\alpha$ and $\beta$ (Figure 3a). Alternatively, one may define areal displacement as the minimum area swept across in a continuous transformation of $\alpha$ to $\beta$ (Figure 3b). Lastly, if regions are marked as being to the right or left of each polyline, then the displacement area may be conceptualized as the total area that is on one side of $\alpha$ but the other side of $\beta$, i.e. the area that "shifts sides" when $\alpha$ is transformed to $\beta$ (Figure $3 \mathrm{c})$.

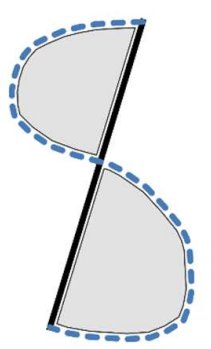

(a)

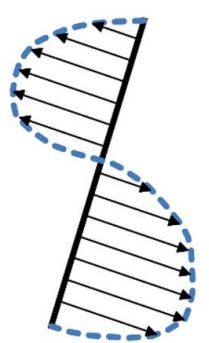

(b)

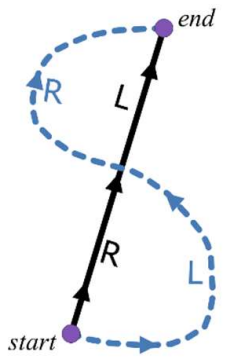

(c)
Figure 3: Three definitions of areal displacement applied to a standard crossing pattern: (a) $\delta_{\text {enclosure, }}$ (b) $\delta_{\text {sweep, }}$ and (c) $\delta_{\text {shift. }}$

We denote these three alternative definitions as $\delta_{\text {enclosure, }}$ $\delta_{\text {sweep }}$, and $\delta_{\text {shift }}$ respectively. Among these, $\delta_{\text {enclosure }}$ seems most equivalent to the concepts of White (1985) and McMaster (1986), while $\delta_{\text {sweep }}$ is the minimum homotopy area in computational topology (Chambers andWang 2013, Fasy et al. 2017). As far as we are aware, $\delta_{\text {shift }}$ has not been previously described.

The preceding definitions all seem intuitive, and in the case of a standard crossing pattern they yield identical results. However, results differ in non-standard patterns. We now discuss the computation of each metric and illustrate the differences between them.

\section{Enclosure}

We formally define enclosure ( $\left.\delta_{\text {enclosure}}\right)$ as the sum of absolute areas of all non-overlapping, simple polygons that are completely enclosed by one or more segment of either polyline (Figure 4). These are the "error polygons" of White (1985), but our definition explicitly includes regions surrounded by segments of just one polyline, as can happen in the case of self-intersection.

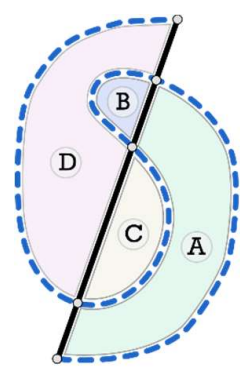

(a)

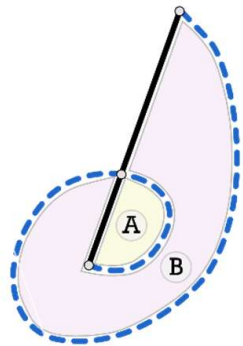

(b)

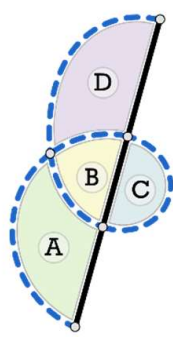

(c)
Figure 4: Enclosure for three non-standard crossing patterns. (a) back-crossing $\left(\delta_{\text {enclosure }}=\mathrm{A}+\mathrm{B}+\mathrm{C}+\mathrm{D}\right),(\mathrm{b})$ enclosed endpoint $\left(\delta_{\text {enclosure }}=\mathrm{A}+\mathrm{B}\right)$, and $(\mathrm{c})$ self-intersection $\left(\delta_{\text {enclosure }}=\mathrm{A}+\mathrm{B}+\mathrm{C}+\mathrm{D}\right)$.

To calculate $\delta_{\text {enclosure }}$ it is first necessary to enumerate the simple error polygons. This can be accomplished with standard computational algorithms, i.e. by (a) identifying all intersections between $\alpha$ and $\beta$ as well as all selfintersections, (b) constructing a graph from the edges between intersections, and (c) enumerating the faces of the graph (de Berg et al. 2008). It is also possible to use existing GIS tools to construct error polygons, and in the case of a standard crossing pattern these tools will produce consistent results. However, this is not true for other crossing patterns. For example, we found that for the back-crossing configuration shown in Figure 4a, ArcGIS produces all four polygons, whereas QGIS only produces $\mathrm{A}$ and $\mathrm{D}$. Therefore, care must be taken when using standard GIS tools on non-standard topological configurations to ensure that the results conform to the intended formal definition of the error metric.

Separate from computational issues, $\delta_{\text {enclosure may be }}$ questioned at a semantic level. As shown in Figure 1, there can be enclosed regions that seem conceptually irrelevant to the concept of displacement. Similarly, if $\alpha$ and $\beta$ are closed polygons (e.g. a lake), then it is usually "obvious" that at least one large internal polygon should be excluded since they represent the interior of the lake in both versions. However, by the same logic sometimes more than one polygon should be excluded from areal displacement (e.g. polygons marked $*$ in Figures 5). In the case of self-intersection there are also cases where one might reasonably conclude that an enclosed polygon should be counted twice. For example, the polygon marked $* *$ in Figure $5 b$ is clearly in the interior of $\alpha$, but its relation to $\beta$ is not as obvious. Arguably, it is located on the exterior of both surrounding line segments and is thus "twice exterior". Thus, caution must be applied when using $\delta_{\text {enclosure }}$ to ensure that the results are semantically meaningful and unambiguous when applied to a particular dataset. For use across multiple datasets, or as a general measure of areal displacement, $\delta_{\text {enclosure }}$ does not seem justifiable in the cases illustrated. 


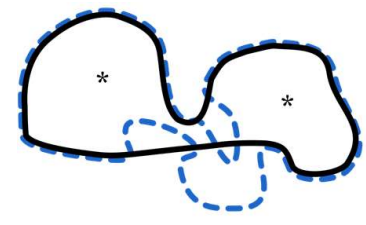

(a)

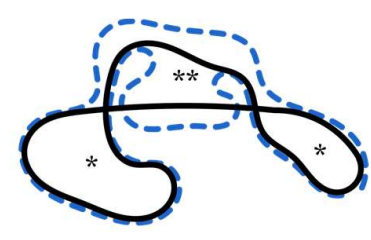

(b)
Figure 5: Configurations where semantic meaning of $\delta_{\text {enclosure }}$ seems questionable: $(*)$ enclosed polygons seem obviously irrelevant to areal displacement, $(* *)$ enclosed polygon should perhaps be counted twice.

\section{Minimum Homotopy Area}

The minimum homotopy area $\left(\delta_{\text {sweep }}\right)$ is the smallest total area of individual homotopy moves required to transform $\alpha$ to $\beta$, where a homotopy move involves sweeping one or more polyline segments across an enclosed polygon (Chambers and Wang 2013, Fasy et al. 2017). This is illustrated in Figure 6. For example, in the back-crossing pattern (Figure 6a), $\delta_{\text {sweep }}$ is $\mathrm{A}+2 \mathrm{~B}+\mathrm{D}$, obtained through the following homotopy moves:

1) $\alpha 2 \rightarrow \beta 3$

2) $[\alpha 3, \beta 3, \alpha 1] \rightarrow \beta 1$

3) $\alpha 4 \rightarrow[\beta 2, \beta 3, \beta 4]$

Note that a transformation from $\alpha$ to $\beta$ could also be achieved by sweeping twice through $\mathrm{C}$ instead of $\mathrm{B}$, but this would result in a larger area. Since the two sequences are topologically identical, this indicates that computation of $\delta_{\text {sweep }}$ is a combinatorial problem that cannot be solved by topological analysis alone. Instead, it requires checking alternative sequences of homotopy moves to find the one that sweeps over the minimum area.

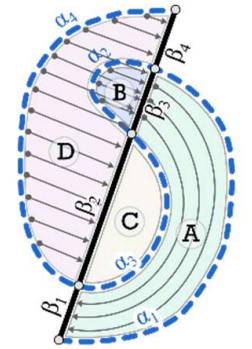

(a)

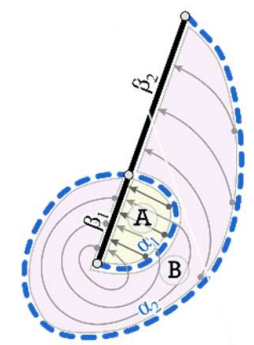

(b)

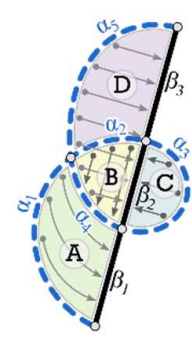

(c)
Figure 6: Minimum homotopy area $\left(\delta_{\text {sweep }}\right)$ for three nonstandard crossing patterns. (a) back-crossing $\left(\delta_{\text {sweep }}=A+2 B+D\right)$, (b) enclosed endpoint $\left(\delta_{\text {sweep }}=2 \mathrm{~A}+\mathrm{B}\right)$, and (c) self-intersection $\left(\delta_{\text {sweep }}=\mathrm{A}+2 \mathrm{~B}+\mathrm{C}+\mathrm{D}\right)$.

Chambers and Wang (2013) present an algorithm for computing $\delta_{\text {sweep }}$ that is robust in the absence of selfintersections on either $\alpha$ or $\beta$. Their algorithm is based on the observation that computation of $\delta_{\text {sweep }}$ is trivial for any subsection of the input polylines for which the error polygons have either consistently non-negative or consistently non-positive winding number (see below). For other cases, they divide the difference polygon into sections with consistent winding number and no backcrossings. Although their algorithm is reasonably straightforward, it is not trivial and does not appear to have been implemented yet (Chambers and Vejdemo-
Johansson 2015), perhaps because a more robust algorithm that can handle self-intersecting input polylines is sought. Fasy et al. (2017) describe an approach for handling self-intersections that involves searching for and processing a broader class of self-overlapping subpolygons, and Evans (2018) presents some simplifying concepts that may facilitate eventual implementation.

The minimum homotopy area appears to be a conceptually robust and intuitive specification of areal displacement, because it captures the essence of displacement as a dynamic process. That is, the transformation of $\alpha$ to $\beta$ through a series of homotopy moves is analagous to the simplification of a polyline in a series of steps, where each homotopy move captures the areal displacement introduced at each step. In a standard crossing pattern (Figure 3), each simple enclosed polygon will be swept across exactly once, so that $\delta_{\text {sweep }}=\delta_{\text {enclosure }}$. In a back-crossing (Figure 6a), at least one interior polygon will need to be swept across twice, but at least one other will not need to be swept across at all, resulting in $\delta_{\text {sweep }} \leq \delta_{\text {enclosure. In the case of an enclosed endpoint }}$ (Figure 6b) or self-intersection (Figure 6c), at least one interior polygon will need to be swept across twice, resulting in $\delta_{\text {sweep }}>\delta_{\text {enclosure }}$.

Unfortunately, an implementation of the minimum homotopy area computation is not yet available. It would be useful if at least of the algorithm by Chambers and Wang (2013) were implemented, but until this becomes available other measures are needed. We now introduce a third measure of areal displacement that shares some desirable properties of minimum homotopy area, but is much simpler to compute.

\section{Shift Displacement}

Imagine an observer traversing first the original polyline and then later the simplified polyline in the same direction. On the second traversal, the observer will notice that some regions observed to the left of the polyline in the first traversal have now shifted to the right, and others have shifted from right to left, as a result of simplification. The regions left and right of each section of each polyline are illustrated in Figure 7 for the three non-standard crossing patterns.

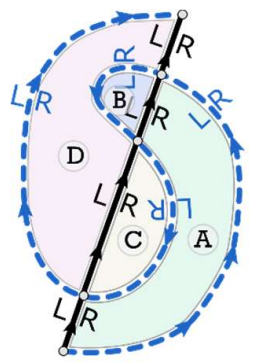

(a)

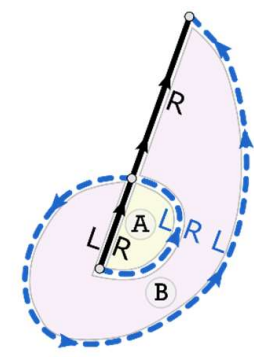

(b)

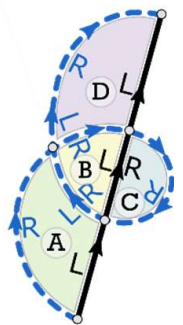

(c)
Figure 7: Left and right relations for three non-standard crossing patterns. (a) back-crossing, (b) enclosed endpoint, and (c) selfintersection.

In the standard and back-crossing patterns, the regions that are left and right of each enclosed polygon can be 
defined unambigously. For example, in Figure 7a, regions A is left of both adjacent segments of $\alpha$, but right of both adjacent segments of $\beta$. As such, it has switched sides and should be included in shift displacement. On the other hand, region B is left of both $\alpha$ and $\beta$ and thus should not be included in shift displacement.

The simple definition above becomes ambiguous in the presence of enclosed endpoints and self-intersections. For example, in Figure $7 \mathrm{~b}$ region $\mathrm{B}$ is left of one segment on $\alpha$ but right of the other, and same is true of $\beta$. Similarly, in Figure $7 \mathrm{c}$ regions A \& D are on both sides of different segments of $\alpha$.

If the notions of left and right are ambiguous, then they must be replaced by similar but unambiguous concepts. A key insight is gained by considering each polyline to be part of the boundary of a larger polygon. To effect this reconceptualization, we construct an arbitrary polyline $\gamma$ connecting the shared end point of $\alpha$ and $\beta$ to their shared start point, and then construct polygon $\alpha^{\prime}=[\alpha+\gamma]$ and polygon $\beta^{\prime}=[\beta+\gamma]$ (Figure 8 ). The boundaries of polygons $\alpha^{\prime}$ and $\beta^{\prime}$ are thus identical everywhere except for the section between the shared start and end points of the original and simplified polylines being evaluated. We then define $\delta_{\text {shift }}$ as the shift in area from the interior of $\alpha^{\prime}$ to the exterior of $\beta^{\prime}$, plus the shift in area from the exterior of $\alpha^{\prime}$ to the interior of $\beta^{\prime}$.

This solution introduces another problem, however, as the cases of enclosed endpoint and self-intersecting polyline both necessarily lead to at least one self-intersecting polygon. We have succeeded in merging these two problems into one, but what, then, are the interior and exterior of a self-intersecting polygon?

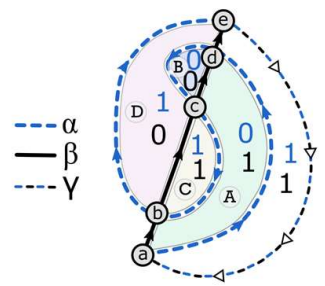

(a)

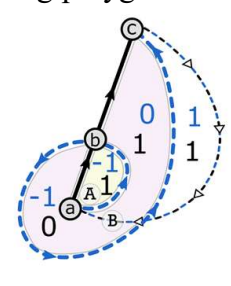

(b)

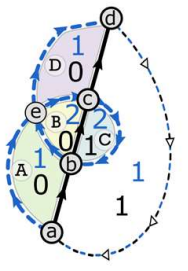

(c)
Figure 8: Shift displacement for three non-standard crossing patterns, calculated as the area of each simple polygon multiplied by its difference in winding number with respect to $\alpha^{\prime}$ and $\beta^{\prime}$ (indicated by numbers within each simple polygon): (a) back-crossing $\left(\delta_{\text {shift }}=A+D\right),(b)$ enclosed endpoint $\left(\delta_{\text {shift }}=2 A+B\right)$, and $(c)$ self-intersection $\left(\delta_{\text {shift }}=A+2 B+C+D\right)$.

To answer this we turn to the well known topology concept of winding number (Figure 9). For any connected subregion $\mathrm{A}$ of a polygon $\mathrm{P}$, the winding number wn $(\mathrm{A}, \mathrm{P})$ is defined as the number of times the boundary of $\mathrm{P}$ winds clockwise around $\mathrm{A}$. Counterclockwise windings are assigned negative winding numbers, while the exterior of the polygon has a winding number of 0 .

The winding number naturally finds its way into many spatial algorithms. For example, a winding number algorithm provides correct results for the point-inpolygon problem in the presence of self-overlapping polygons (Sunday 2012). Most notably for our purposes, if the standard surveyor's formula is used to compute the area of a self-intersecting polygon, the computed area will equal the sum of the areas of each subregion times their winding number (Courant 1934, pp. 311-314). This makes sense when one considers a continuous transition from an ordinary polygon (i.e. a single region with winding number $=1$ ) to one with subregions with winding numbers of $2,-1$, etc. For example, a region in which a polygon overlaps itself is assigned a winding number of 2 . This can be interpreted as indicating that the region is covered twice by the polygon. In fact, the winding number of a region within a polygon is also sometimes referred as its density (Grünbaum and Shephard 1990).

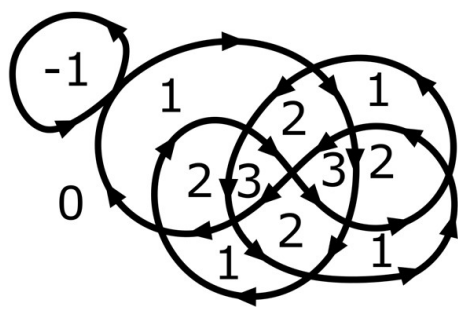

Figure 9: Illustration of the winding number for sections of a self-intersecting polygon.

This observation leads to a natural definition of $\delta_{\text {shiftlq }}$ as the sum of the absolute areas of simple non-overlapping enclosed polygons, multiplied by the differences in their winding numbers in $\alpha^{\prime}$ and $\beta^{\prime}$. This can be expressed by the following formula:

$$
\delta_{\text {shift }}(\alpha, \beta)=\sum_{p \in P}\left|w n\left(p, \alpha^{\prime}\right)-w n\left(p, \beta^{\prime}\right)\right| \times \operatorname{area}(p)
$$

where $P$ is the set of enclosed polygons.

In a standard crossing pattern, all enclosed polygons will have differences in winding number of -1 or +1 , so that $\delta_{\text {shift }}=\delta_{\text {enclosure. }}$ Returning to the examples in Figure 8 , for the back-crossing pattern (Figure 8a) we see that the formal definition yields the same result as our informal definition based on left/right relations, with regions $B$ and $C$ excluded because both $w n\left(B, \alpha^{\prime}\right)=w n\left(B, \beta^{\prime}\right)$ and also $w n\left(C, \alpha^{\prime}\right)=w n\left(C, \beta^{\prime}\right)$. Thus, for back-crossings we can say generally that $\delta_{\text {shitt }}<\delta_{\text {enclosure. In the case of an enclosed }}$ endpoint (Figure 8b) or self-intersection (Figure 8c), at least one interior polygon will have a difference in winding numbers of 2 or more, resulting in $\delta_{\text {shift }}>$ $\delta_{\text {enclosure. }}$

Note that while the choice of $\gamma$ will influence the way that error polygons are partitioned, it will not influence the difference in winding number at any location, and therefore will not influence the value of $\delta_{\text {shift. This }}$ is because the winding number of a point can be determined by counting the number of right-to-left and left-to-right crossings from that point to infinity in any direction (Grünbaum and Shephard 1990). Since $\gamma$ is shared by $\alpha^{\prime}$ and $\beta$ ', it will contribute the same number of R-L and L-R crossings from any given location, and thus have the same influence on winding number. 


\section{Algorithm to Calculate $\boldsymbol{\delta}_{\text {shift }}$}

Although the addition of the arbitrary polyline $\gamma$ is helpful to illustrate the meaning of $\delta_{\text {shift }}$, it is not necessary for its calculation. Instead, calculation can be performing on the (self-intersecting) difference polygon created by appending $\beta$ (in reverse) to $\alpha$. The general strategy is to detect and insert self-intersections on the difference polygon, and then to use the standard shoelace formula to calculate the area of the difference polygon by summing the trapezoid under each line segment, but with the modification that the sign of the summed term is reversed whenever the winding number on the left becomes negative.

\subsection{Detecting Intersections}

To keep track of winding numbers during traversal, it is necessary to identify and insert vertices at selfintersections. This process is not trivial, so some discussion is warranted. Algorithms to detect selfintersection generally depend on pairwise analysis, i.e. they look at each pair of segments $i$ and $j$ independently. Efficient sweep algorithms avoid performing this test on every segment pair (de Berg et al. 2008), but they still employ a basic pairwise intersection test at their core. Problems can occur, however, in the case of selfosculatory and overlapping polygons (Grünbaum and Shephard 1990; Figure 10). A self-osculatory polygon is one in which at least one vertex lies directly on a segment to which it does not belong (e.g. vertices 4,7 \& 11 in Figure 10). Similarly, an overlapping polygon is one in which an extended portion of one segment lies coincident with another segment (e.g. segments[2,3], [5,6], [13,14] and $[19,20]$ in Figure 10).

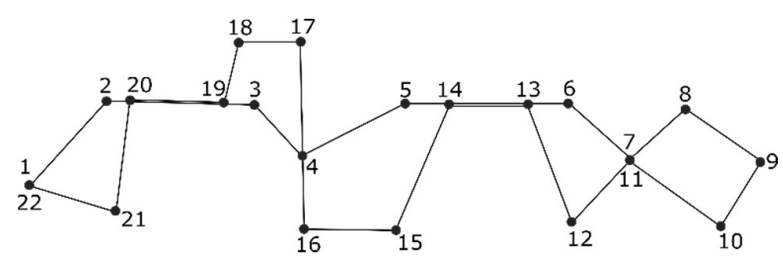

Figure 10: Special cases that must be handled when detecting self-intersections.

Self-osculation and overlap will be especially prevalent in difference polygons formed from the chaining together of two versions of the same polyline, as is the case in line simplification. This is because any segment that escapes alteration during the simplification process will automatically overlap its original counterpart, and any unaltered vertex whose neighboring vertices are altered will form a self-osculation.

The reason why self-osculatory and overlapping polygons create problems for topological analysis is that in these cases, more than two segments are required to determine if an intersection truly occurs or not. Nevertheless, it is possible to identify self-intersections necessary for calculating $\delta_{\text {shift }}$ and other areal properties correctly through pairwise analysis, so long as one accepts that two intersections might be inserted where none are really needed. For example, we will get correct results if the segment [5,6] and segment chain $[12,13,14,15]$ in Figure
10 are found not to intersect at all, but we will also get correct results if they are found to intersect twice at the locations of vertices 13 and 14 . In the latter case, an implicit zero-area region is created between vertices 13 and 14 , but this will not affect our area calculations.

Such cases should be regularly handled in GIS overlay operations such as union and intersection, but we are not aware of any public description of a robust handling procedure so we provide one here. When comparing two line segments, let us refer to the lower id segment as the baseline and the higher id segment as the crossing segment. We first pre-process the difference polygon to remove any sequential coincident vertices. Then, for each candidate pair of segments, the following rules are used to determine if they are considered to intersect:

1) If the baseline start vertex lies exactly on the crossing segment, there is no intersection

2) If the baseline end vertex lies exactly on the crossing segment, extend the baseline to include the next sequential vertex

3) An intersection occurs if the two crossing segment vertices are on different sides ( $\mathrm{R}$ or $\mathrm{L}$ ) of the baseline

4) If a crossing segment vertex lies exactly on the baseline, it is considered to lie to the right of the baseline

The result of applying these rules to the difference polygon in Figure 10 is shown in Table 1. A total of two intersections are found, one in the region of overlap between $[2,3]$ and $[18,19,20,21]$ and the other on the selfosculation between $[3,4,5]$ and $[16,17]$. No intersections are found between $[5,6]$ and $[12,13,14,15]$ or between $[6,7,8]$ and $[10,11,12]$. To test symmetrical cases, the same rules were applied to the vertically flipped image of Figure 10. The results are similar, but with two intersections instead of zero between $[5,6]$ and $[12,13,14,15]$, and also between $[6,7,8]$ and $[10,11,12]$.

\begin{tabular}{|c|c|c|c|c|c|c|c|c|}
\hline B1 & B2 & B1 on X? & B2 on X? & B3 & X1 & X2 & Int? & Flip Int? \\
\hline 2 & 3 & & & & $18(\mathrm{~L})$ & $19(\mathrm{R})$ & yes & no \\
\hline 2 & 3 & & & & $19(\mathrm{R})$ & $20(\mathrm{R})$ & no & no \\
\hline 2 & 3 & & & & $20(\mathrm{R})$ & $21(\mathrm{R})$ & no & yes \\
\hline 3 & 4 & & yes & 5 & $16(\mathrm{R})$ & $17(\mathrm{~L})$ & yes & yes \\
\hline 4 & 5 & yes & & & 16 & 17 & no & no \\
\hline 5 & 6 & & & & $12(\mathrm{R})$ & $13(\mathrm{R})$ & no & yes \\
\hline 5 & 6 & & & & $13(\mathrm{R})$ & $14(\mathrm{R})$ & no & no \\
\hline 5 & 6 & & & & $14(\mathrm{R})$ & $15(\mathrm{R})$ & no & yes \\
\hline 6 & 7 & & yes & 8 & $10(\mathrm{R})$ & $11(\mathrm{R})$ & no & yes \\
\hline 6 & 7 & & yes & 8 & $11(\mathrm{R})$ & $12(\mathrm{R})$ & no & yes \\
\hline 7 & 8 & yes & & & 10 & 11 & no & no \\
\hline 7 & 8 & yes & & & 11 & 12 & no & no \\
\hline
\end{tabular}

Table 1: Example of rule-based intersection test applied to candidate segment pairs in Figure 10. Each candidate pair of baseline B (B1-B2) and crossing line X (X1-X2) is assessed as follows: (a) if $B 1$ is on $X$, there is no intersection, (b) if $B 2$ is on $\mathrm{X}, \mathrm{B}$ is extended to the next vertex (B3), (c) vertices X1 and $\mathrm{X} 2$ are marked as either right or left of $\mathrm{B}$, with vertices exactly on $\mathrm{B}$ marked as right of $\mathrm{B},(\mathrm{d})$ an intersection occurs if $\mathrm{X} 1$ and $\mathrm{X} 2$ are marked as being on opposite sides of $\mathrm{B}$. The last column applies the same test to the vertically flipped image of Figure 10 . 


\subsection{Tracking Winding Number}

Once the difference polygon is constructed and vertices are inserted at intersections, the value of $\delta_{\text {shift }}$ can be computed much more simply than $\delta_{\text {sweep }}$ because there are no permutations that need to be evaluated and compared. In fact, the difference polygon captures all areas that have shifted, and the winding number of each subregion in this polygon is exactly equal to the difference in winding numbers between the same subregion in $\alpha^{\prime}$ and $\beta^{\prime}$.

As a result, $\delta_{\text {shift }}$ can be calculated with a simple modification to the standard "shoelace" algorithm used to compute polygon area. Recall that this algorithm automatically multiplies the area of each sub-region by its winding number (Courant 1934). Modification is necessary to distinguish between regions with positive and negative winding area, which would cancel each other out using the standard formula. To prevent this, we simply reverse the sign when the winding number is negative. That is, we first identify a line segment on the outer boundary with known winding number to its right, and then keep track of the winding number to the right as we traverse the polygon boundary. The simple rule is that if a segment has a winding number $\mathrm{w}$ before an intersection, then the winding number after the intersection is $w+1$ if the intersecting segment crosses from right to left, otherwise it is $\mathrm{w}-1$ (Figure 11; Grünbaum and Shephard 1990). For any segment for which this winding number is less than one, we multiply the corresponding term in the shoelace formula by -1 .
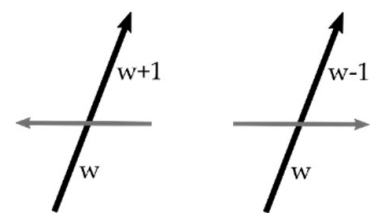

Figure 11: Tracking winding number on the right side while traversing the polygline.

\subsection{Complete Algorithm}

Combining the above observations, a complete algorithm for calculating $\delta_{\text {shift }}$ is as follows:

function $\delta \_$shift $(\alpha, \beta$ w/ shared endpoints):

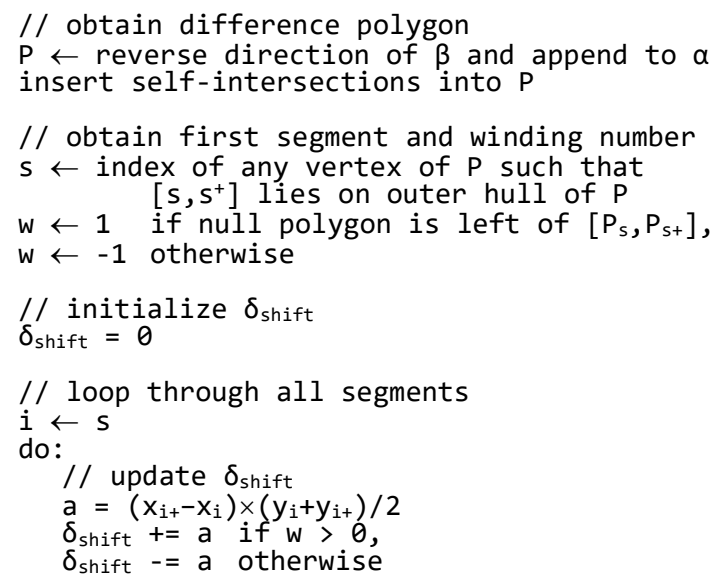

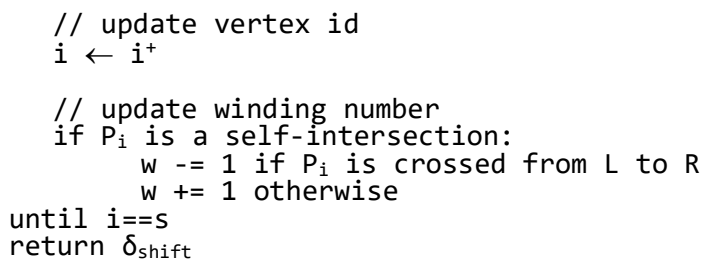

where $P_{i}$ is the vertex on $P$ with index $i, x_{i}$ and $y_{i}$ are the coordinates of $\mathrm{P}_{i}$, and $i^{+}$is the index of the next vertex in sequence along $P$ after $P_{i}, i . e . i^{+}=i+1$ unless $P_{i}$ is the last vertex of $P$, in which case $i^{+}=1$.

\section{Discussion}

Despite its status as a primary quantitative measure of error in line simplification, the concept of areal displacement has not been formally defined within the cartographic community. This paper identifies three distinct semantic definitions of areal displacement and illustrates that they can sometimes lead to dramatially different values. Most notably, it is demonstrated that the naïve method of calculating the total area of enclosure leads to potentially misleading values, especially when the original and simplified polylines conspire to isolate a large region by bridging a narrow gap that connects that region with the exterior of both polylines, either due to a back-crossing or a self-intersection. Additionally, the naïve measure of enclosure fails to double count regions that are doubly displaced, either due to an enclosed endpoint or a self-intersection. These problems are resolved by the topological definition of minimum homotopy area, which we consider semantically to be very appealing, but tools for its computation are not currently available.

A third definition of areal displacement, referred to as shift displacement, is introduced in this paper and shown to be a practical alternative to existing measures. Conceptualized informally as the area that shifts to the left or right during simplification, it is defined formally in terms of changes in the measured areas of a presumed polygon on either side of the polyline being simplified. As such it seems semantically appropriate when the effects of simplification on area measurement are important, or when one wishes to quantify the total area affected by the simplification process.

Even in cases in which minimum homotopy area seems more appropriate semantically, we observe that shift displacement appears to provide a better approximation of minimum homotopy area than enclosure in most cases. In particular, shift displacement avoids the misleading inclusion of large enclosed polygons mentioned above and illustrated in Figure 1 and Figure 5a, and also double-counts most regions of double-overlap created by enclosed endpoints and self-intersections, as illustrated in Figure 5b. On the other hand, shift displacement does not include some regions that must be swept through twice in a continuous transformation from the original to the simplified polyline, such as the smaller "empty" region of a back-crossing (e.g. polygon B in Figures 1 and 7a) as 
well as some instances of overlap created by more complex self-intersection scenarios (not shown).

Although this paper illustrated several non-standard topological configurations that pose challenges for defining areal displacement, it remains unknown how often such configurations occur in real-world scenarios, and how severe the effects are on the three metrics described here. Self-intersections can be avoided with some simplification algorithms, but this comes with a cost in terms of computation time. Back-crossings and enclosed endpoints do not violate any logical constraint, so it must be assumed they they can occur in any given setting. Further research is required to assess empirically the frequency of non-standard patterns and their influence on measures of areal displacement.

While this paper presents some quantitative measures of areal displacement for use in evaluating simplified line features, cartographic generalization has multiple aims, and quantitative assessment of error should be used appropriately in conjunction with visual assessment methods (Stoter et al. 2014). In many situations, quantitative metrics of fidelity will be of secondary importance to shape recognition and simplicity, as illustrated for example by recent increased interest in schematization (Mackaness and Reimer 2014). On the other hand, cartographic generalization is also an integral part of data modeling and data dissemination by national mapping agencies (Weibel and Dutton 1999), and there has been increased interest in preserving line characteristics for specific analytical purposes, such as hydrologic analyses (Stanislawski et al. 2015).

When a single quantitative error measure is needed to assess the degree of distortion introduced by line simplification, areal displacement should be considered a foundational measure. Unlike the Hausdorff and Frechet distances which measure the maximum distortion, areal displacement provides a measure of average distortion when divided by the length of the original or simplified line (Veregin 2000). In these cases, our analysis suggests that minimum homotopy area should be considered as best capturing the general notion of "displacement", but that shift displacement should be preferred over enclosure due to its similarity to minimum homotopy area and its semantic appeal in capturing changes in coverage of adjacent regions.

Note: A robust open source python implementation of shift displacement will be made available prior to publication.

\section{References}

Chang, Hsien-Chih and Erickson, Jeff. 2017. Untangling Planar Curves. Discrete \& Computational Geometry, 58:889-920.

Chambers, E.W. and Wang, Y. (2013). Measuring similarity between curves on 2-manifolds via homotopy area. SoCG 2013, Rio de Janeiro, Brazil, June 17-20.

Chambers, E.W. and Vejdemo-Johansson, M. (2015). Computing minimum area homologies. Computer Graphics Forum, 34(6):13-21.
Courant, R. (1934). Differential \& Integral Calculus, Volume I. London: Blackie \& Son Ltd.

de Berg, M., Cheong, O., van Kreveld, M. and Overmars, M. (2008). Computational Geometry: Algorithms and Applications (third edition). Berlin: Springer-Verlag.

Evans, P. (2018). On self-overlapping curves, interior boundaries, and minimum area homotopies. Thesis: Department of Mathematics, Tulane University.

Fasy, B.T., Karakoç, S. and Wenk, C. (2017). On minimum area homotopies of normal curves in the plane. Pre-print, July 2107. arXiv:1707.02251.

Grünbaum, B. and Shephard, G.C. (1990). Rotation and winding numbers for planar polygons and curves. Transactions of the American Mathematical Society, 322(1):169-187.

Lee, D. and Hardy, P. (2005). Automating Generalization - Tools and Models. Proceedings of the 22nd International Cartographic Conference (ICC 2005), A Coruña, Spain, July 11-16.

Mackaness, W. and Reimer, A. (2014). Generalisation in the context of schematised maps. In Burghardt, D., Duchéne, C. and Mackaness, W., editors, Abstracting Geographic Information in a Data Rich World, ch. 9. Lecture notes in geoinformation and cartography. Heidelberg: Springer.

McMaster, R.B. (1986). A statistical analysis of mathematical measures for linear simplification. The American Cartographer, 13(2):103-116.

Méneroux, Y, Le Guilcher, A., Orfila, O., Lusetti, B., Saint Pierre, G. and Mustière, S. 2017. Using surrogate road network for map-matching. A sensitivity analysis of positional accuracy. Geocomputation 2017.

Muller, J.-C. (1987). Fractal and automated line generalization. The Cartographic Journal, 24(1):27-34.

Raposo, P. (2013). Scale-specific automated line simplification by vertex clustering on a hexagonal tesselation. Cartography and Geographic Information Science, 40(5):427-443.

Saalfeld, A. (1999). Topologically consistent line simplification with the Douglas-Peucker algorithm. Cartography and Geographic Information Science, 26(1):7-18.

Shen, Y., Ai, T. and He, Y. (2018). A new approach to line simplification based on image processing: A case study of water area boundaries. International Journal of Geo-Information, 7(2):41.

Stanislawski, L., Buttenfield, B. and Doumbouya, A. (2015). A rapid approach for automated comparison of independently derived stream networks. Cartography and Geographic Information Science, 42(5):435-448.

Stanislawski, L., Kronenfeld, B., Buttenfield, B. and Brockmeyer, T. (2018). Generalizing linear stream features to preserve sinuosity for analysis and display: A pilot study in multi-scale data science. AutoCarto/UCGIS 2018 Proceedings, Madison, Wisconsin, pp. 111-119. 
Stoter, J., Zhang, X., Stigmar, H. and Harrie, L. (2014). Evaluation in generalisation. In Burghardt, D., Duchéne, C. and Mackaness, W., editors, Abstracting Geographic Information in a Data Rich World, ch. 9. Lecture notes in geoinformation and cartography. Heidelberg: Springer.

Sunday, D. (2012). Inclusion of a point in a polygon. http://geomalgorithms.com/a03-_inclusion.html (last accessed Dec. 2, 2018).

Weibel, R. and Dutton, G. (1999). Generalising spatial data and dealing with multiple representations. In Longley, P., Goodchild, M., Maguire, D. and Rhind, D. Geographical Information Systems, Volume 1: Principles and Technical Issues, ch. 10. New York: John Wiley \& Sons.

White, E. (1985). Assessment of line-generalization algorithms using characteristic points. The American Cartographer, 12(1):17-27.

Veregin, H. (2000). Quantifying positional error induced by line simplification. International Journal of Geographical Information Science, 14(2):113-130.

Visvalingam, M. and Whyatt, J.D. (1990). The DouglasPeucker algorithm for line simplification: Re-evaluation through visualization. Computer Graphics Forum, 9(3):213-228.

Visvalingam, M. and Whyatt, J.D. (1993). Line generalisation by repeated elimination of points. The Cartographic Journal, 30(1):46-51. 